\title{
An Inventory Model with Stock-Dependent Demand Rate and Maximization of the Return on Investment
}

\author{
Valentín Pando ${ }^{1,2, *(\mathbb{D})}$, Luis A. San-José ${ }^{3,4}$ (D) and Joaquín Sicilia 5 (D) \\ 1 Departamento de Estadística e Investigación Operativa, Universidad de Valladolid, 47002 Valladolid, Spain \\ 2 IUFOR-Instituto Universitario de Investigación en Gestión Forestal Sostenible (UVa-INIA), \\ 34004 Palencia, Spain \\ 3 IMUVA-Instituto de Investigación en Matemáticas, Universidad de Valladolid, 47002 Valladolid, Spain; \\ augusto@mat.uva.es \\ 4 Departamento de Matemática Aplicada, Universidad de Valladolid, 47002 Valladolid, Spain \\ 5 Departamento de Matemáticas, Estadística e Investigación Operativa, Universidad de La Laguna, \\ 38200 San Cristóbal de La Laguna, Spain; jsicilia@ull.es \\ * Correspondence: vpando@uva.es
}

Citation: Pando, V.; San-José, L.A.; Sicilia, J. An Inventory Model with Stock-Dependent Demand Rate and Maximization of the Return on Investment. Mathematics 2021, 9, 844. https://doi.org/10.3390/ math9080844

Academic Editor: Massimiliano Ferrara

Received: 12 March 2021

Accepted: 11 April 2021

Published: 13 April 2021

Publisher's Note: MDPI stays neutral with regard to jurisdictional claims in published maps and institutional affiliations.

Copyright: (C) 2021 by the authors Licensee MDPI, Basel, Switzerland. This article is an open access article distributed under the terms and conditions of the Creative Commons Attribution (CC BY) license (https:/ / creativecommons.org/licenses/by/ $4.0 /)$.

\begin{abstract}
This work presents an inventory model for a single item where the demand rate is stockdependent. Three fixed costs are considered in the model: purchasing cost, ordering cost and holding cost. A new approach focused on maximizing the return on investment (ROI) is used to determine the optimal policy. It is proved that maximizing profitability is equivalent to minimizing the average inventory cost per item. The global optimum of the objective function is obtained, proving that the zero ending policy at the final of a cycle is optimal. Closed expressions for the lot size and the maximum ROI are determined. The optimal policy for minimizing the inventory cost per unit time is also obtained with a zero-order point, but the optimal lot size is different. Both solutions are not equal to the one that provides the maximum profit per unit time. The optimal lot size for the maximum ROI policy does not change if the purchasing cost or the selling price vary. A sensitivity analysis for the optimal values regarding the initial parameters is performed by using partial derivatives. The maximum ROI is more sensitive regarding the selling price or the purchasing cost than regarding the other parameters. Some useful managerial insights are deduced for decision-makers. Numerical examples are solved to illustrate the obtained results.
\end{abstract}

Keywords: EOQ models; return on investment maximization; stock-dependent demand rate; minimizing average inventory cost per item

\section{Introduction}

The traditional literature on inventory models assumes that the demand of the items is uniform on time, and independent of the levels of stock for sale in the warehouse. However, for some types of products, it is a proven fact that the quantity in stock can influence the item sales. Low stocks of certain items make customers feel that they are not fresh and drastically reduce sales of those items. Conversely, increasing the amount of the article displayed for sale induces more consumers to buy it. This probably occurs owing to the increase in the visibility of the items. Hence, inventory managers have observed that the demand rate may be influenced by the stock levels. Thus, in inventory control, the possible effect of the inventory level on the demand has been recognized and studied by some researchers. As a starting point, Wolfe [1] showed empirical evidence that sales of style merchandise are almost proportional to the displayed inventory. In addition, the book by Levin et al. [2] mentioned that the presence of inventory has a motivational effect, as evidenced by the issue that large piles of goods displayed in a supermarket will lead the customers to buy more. Later, Silver and Peterson [3] confirmed the results obtained by Wolfe that sales at the retail level tend to be directly proportional to the displayed stock. Larson and DeMarais [4] investigated 
the impact of displayed inventory on sales for four health and beauty items and offered possible explanations for the effect of stock level on demand. They introduced the term "psychic shock" to refer to the reasons for this effect. Achabal et al. [5] provided empirical evidence that displayed inventory increases the demand of goods. The adventage of using a stock-dependent demand was highlighted by Balakrishnan et al. [6]. In addition, Koschat [7] analyzed a real case in the magazine industry, proving that demand can indeed vary with inventory level. Thus, an inventory drop for one brand leads to a decrease of demand for that brand and in an increase of demand for a competing brand. Next, we present a revision of the literature on inventory models with this type of demand.

Baker and Urban [8] developed a first deterministic inventory system for a stockdependent demand rate. Padmanabhan and Vrat [9] analyzed an inventory system for deteriorating multi-items with stock-dependent demand. Datta and Pal [10] established the optimal inventory policy for a system where the demand rate is constant if the inventory level is less than a given level, and this rate depends on the instantaneous inventory level when it is greater than that given level. Later, Urban [11] relaxed the condition of zero-inventory at the end of the inventory cycle considered by Datta and Pal [10]. The model of Baker and Urban [8] was generalized by Pal et al. [12] for items with a constant deterioration rate. Giri et al. [13] also extended the inventory model of Urban [11] in the same way. Giri and Chaudhuri [14] developed the economic order quantity model for stockdependent demand considering a non-linear holding cost. Datta and Paul [15] studied a multi-period inventory system where the demand rate is stock-dependent and sensitive to the selling price. Ouyang [16] introduced a model with stock-dependent demand for deteriorating items under conditions of inflation and time-value of money, considering the present value of the total inventory cost as the objective function. Chang [17] analyzed an inventory model with non-linear holding cost, where the demand rate depended on the stock level. Later, Pando et al. [18-20] considered three models with maximization of the profit per unit time and stock-dependent demand, where the holding cost was non-linear on time, on the stock level or, even more, on both quantities. Yang [21] presented an inventory model where the demand rate and the holding cost rate are stock-dependent, and partially backlogged shortages. Annadurai and Uthayakumar [22] described a lot-sizing model for deteriorating items with stock-dependent demand, partially backordered shortages and delay in payments. In the same line, Choudhury et al. [23] analyzed an inventory system with shortages and time-varying holding costs.

In all the aforementioned works, the objective was either to minimize the total cost related to the administration of the inventory or to maximize the profit or gain obtained with the sale of the items. The comparison between these two alternatives leads to observe that the solution of the minimum inventory cost carries a low profit, while the maximum profit solution carries high inventory costs. In view of this, perhaps the inventory manager may prefer a solution that provides a high profit without greatly increasing the total cost invested in the inventory. Then, the maximization of the ratio between the profit and the total cost of the inventory system, i.e., the return on investment (ROI), could be more interesting. Indeed, if the inventory manager can invest in different products, it would seem reasonable to select the aim that provides a higher return on investment. This is the approach used in this paper.

In the literature on inventory models, there are several papers dedicated to analyzing inventory systems with the maximization of return on investment. Thus, one of the first attempts in adapting the EOQ model to the objective of ROI maximization was made by Raymond [24]. Schroeder and Krishnan [25] studied an inventory system with the aim of return on investment maximization. They also enumerate the conditions under which ROI is an appropriate criterion and contrast it to the traditional cost minimization and profit maximization criteria. Morse and Scheiner [26] analyzed cost minimization, return on investment (ROI) and residual income as alternative criteria and investment measuring for inventory models. Arcelus and Srinivasan [27] proposed an economic 
ordering quantity model for items with price-dependent demand, in which the goal was to find the lot size and the selling price that maximize the return on the funds tied up in inventory. Later, Arcelus and Srinivasan [28] developed efficient policies for inventory systems with price-dependent demand under various optimizing criteria. In addition, Giri [29] studied a stochastic inventory model with price-dependent demand where the goal is to maximize the expected utility of the net present value of the earnings over an infinite planning horizon. Jordan [30] presented a comparative study of the effectiveness of several dynamic lot-sizing methods with respect to return on investment. Rosenberg [31] analyzed and compared the criteria profit maximization and return on investment maximization, considering logarithmic demand functions. In addition, Calderón-Rossell [32] studied the relationship between the internal rate of return and the return on investment as criteria for evaluating the profitability of an investment. The maximization of the return on investment in an EOQ model was also considered by Trietsch [33]. He devised the term ROQ to denote the ROI-maximizing solution and proved that ROQ is bounded from above by the EOQ formula. Otake et al. [34] and Otake and Min [35] also studied inventory and investment in quality improvement policies to maximize return on investment. Li et al. [36] analyzed a return on investment (ROI) maximization model in an inventory with capital investment in setup and quality operations. Protopappa-Sieke and Seifert [37] developed a mathematical model that determines the optimal order quantity under working capital restrictions and payment delays. Choi and Chiu [38] studied the fashion retailer's inventory decision making problem as a news-vendor problem and proposed measuring the sustainability of the fashion retailer from various perspectives, including the expected amount of left-over, the expected sales to expected goods leftover ratio, the rate of return on investment, and the probability of meeting the profit target. Hidayat and Fauzi [39] developed an algorithm to determine the optimal procurement decision on a pharmacy inventory system for items with expired date.

The consideration of a stochastic demand has been widely used in inventory theory. In this case, risk management objectives must be analyzed. The conditional value at risk (CVaR) has been used, for example, by Wang et al. [40], or Tao et al. [41]. Furthermore, stochastic demand allows considering more realistic inventory models within a supply chain (see, for example, Ullah et al. [42]), or within a manufacturing production system, where a work-in-process inventory during the production run is necessary (see, for example, Dey et al. [43]). However, to the best of our knowledge, stochastic inventory models whose objective is the maximization of the return on investment have not been analyzed in the literature on inventory. Therefore, we have not addressed this issue, and we propose it as a possible research line in the future.

In this work, the optimal inventory policy for a system with stock-dependent demand rate is analyzed, where the aim is the maximization of the return on investment. Taking into account the total cost and the profit (calculated as the difference between revenue and cost) obtained in the inventory, the objective is to determine the optimal inventory policy that maximizes the ratio profit/cost. Even more, the optimal solution which minimizes the inventory cost per unit time for the system is also examined. In such a way, the comparison between both optimal solutions allows us to analyze the likeness and the differences between the maximum return on investment policy and the classical policy of minimum cost per unit time that has been mostly used in the inventory theory. In this research line, Pando et al. [44] found the solution that maximizes the profitability ratio in an inventory model with non-linear holding cost, but the problem with a minimum cost per unit time is not addressed and the comparison between both solutions is not studied. Other papers on this subject are Ishfaq and Bajwa [45] and Pando et al. [46].

To illustrate the similarities and differences between the model analyzed in this paper and the cited publications with a bigger link to this work, in Table 1, we show a classification of these papers according to the type of demand, the structure of the holding cost, and the studied objective. As you can see, the model analyzed in this paper is the only one with stock-dependent demand rate which compares the problems of maximum ROI and 
minimum cost per unit time. The two approaches could be interesting. For example, non-profit inventory systems, where the goal is not to make a profit but to provide a service to customers (for example, inventory systems for humanitarian aids items), may prefer to minimize the inventory cost per unit time. On the other hand, if the objective is to obtain a profit with the lowest amount of money, the solution with the maximum return investment will be preferred. The comparison between both policies could get out interesting highlights in the inventory management.

The script of the paper is as follows. In Section 2, the statement of the model is proposed, after setting the notation and the basic assumptions. In Section 3, some properties are discussed, and the ROI optimal solution is provided. In addition, the optimal policy that minimizes the inventory cost per unit time is determined, and the differences between both solutions are commented. Section 4 presents a sensitivity analysis for the optimal order quantity and the maximum return on investment regarding the input parameters of the system. In Section 5, numerical examples are presented to illustrate the theoretical results. Finally, the conclusions are drawn and future research lines are addressed in Section 6.

Table 1. Major characteristics of the main publications on inventory models cited in the Introduction.

\begin{tabular}{|c|c|c|c|}
\hline Paper & Demand & Holding Cost & Objective \\
\hline Baker and Urban [8] & stock-dependent & constant & maximum profit \\
\hline Padmanabhan and Vrat [9] & stock-dependent & constant & maximum profit \\
\hline Datta and Pal [10] & stock-dependent & constant & minimum cost \\
\hline Urban [11] & stock-dependent & constant & maximum profit \\
\hline Pal et al. [12] & stock-dependent & constant & minimum cost \\
\hline Giri et al. [13] & stock-dependent & constant & minimum cost \\
\hline Giri and Chaudhuri [14] & stock-dependent & $\begin{array}{l}\text { time-dependent or } \\
\text { stock-dependent }\end{array}$ & minimum cost \\
\hline Datta and Paul [15] & stock-dependent & constant & maximum profit \\
\hline Ouyang et al. [16] & stock-dependent & constant & minimum cost \\
\hline Chang [17] & stock-dependent & $\begin{array}{l}\text { time-dependent or } \\
\text { stock-dependent }\end{array}$ & maximum profit \\
\hline Pando et al. [18] & stock-dependent & time-dependent & maximum profit \\
\hline Pando et al. [19] & stock-dependent & stock-dependent & maximum profit \\
\hline Pando et al. [20] & stock-dependent & time and stock-dependent & maximum profit \\
\hline Yang [21] & stock-dependent & time-dependent & maximum profit \\
\hline Choudhuri et al. [23] & stock-dependent & time-dependent & maximum profit \\
\hline Arcelus and Srinivasan [27] & price-dependent & constant & maximum ROI \\
\hline Trietsch [33] & constant & constant & maximum ROI \\
\hline Otake et al. [34] & constant & constant & maximum ROI \\
\hline Otake and Min [35] & constant & constant & maximum ROI \\
\hline Li et al. [36] & constant & constant & maximum ROI \\
\hline Pando et al. [44] & stock-dependent & time and stock-dependent & maximum ROI \\
\hline Pando et al. [46] & stock-dependent & time dependent & maximum ROI \\
\hline This paper & stock-dependent & constant & $\begin{array}{l}\text { maximum } \mathrm{ROI} \text { and } \\
\text { minimum cost }\end{array}$ \\
\hline
\end{tabular}

\section{Model Statement}

The inventory system considered in this paper is designed for a single item with an infinite planning horizon. A continuous review of inventory is supposed, considering instantaneous replenishment, and shortages are not allowed. The unit purchasing cost $p$ and the unit selling price $v$ are fixed parameters. The ordering cost per order $K$, and the holding cost per unit and per unit time $h$, are also fixed parameters.

On the other hand, the demand rate at time $t$ is a known function $D(t)$, which depends on the inventory level $I(t)$ at each time. As in Baker and Urban [8], this function is given 
by $D(t)=\lambda[I(t)]^{\beta}$, with $\lambda>0$ and $0 \leq \beta<1$. Then, the demand rate decreases over time as the inventory level decreases. The scale parameter $\lambda$ of this function can be considered as the number of potential customers per unit time. The $\beta$ exponent is a measure of the responsiveness of the demand rate to changes in the inventory level. It is known as the elasticity of the demand rate regarding the inventory level. Note that, if $\beta=0$, this function leads to the basic model with constant demand rate.

An interesting property of this demand function is that the replenishment of the inventory before the stock is depleted leads to an increase in the demand rate, and therefore an increase in sales revenue. Then, the higher ordering and holding cost could offset and the profit could be improved. For example, Baker and Urban [8] found that the maximum profit per unit time in an inventory model with this demand function is obtained with a non-zero order point.

For this reason, this model considers two decision variables: the order level $S$ (inventory level at the beginning of the inventory cycle), and the order point $s$ (inventory level when the inventory is replenished). In this way, the lot size is $q=S-s$. Obviously, $S=\lim _{t \rightarrow 0^{+}} I(t)=I(0+)$, and the length of the inventory cyle $T$ can be evaluated with the equation $I(T-)=\lim _{t \rightarrow T^{-}} I(t)=s$.

Table 2 presents the notation used in the paper.

Table 2. Notation for the model.

\begin{tabular}{ll}
\hline$S$ & order level $(S>0)$ \\
$s$ & order point $(0 \leq s<S)$ \\
$q$ & lot size, $q=S-s(>0)$ \\
$T$ & length of an inventory cycle $(>0)$ \\
$t$ & elapsed time in the inventory $(\leq T)$ \\
$I(t)$ & level of the inventory at time $t(s \leq I(t) \leq S)$ \\
$p$ & unit purchasing cost $(>0)$ \\
$v$ & unit selling price $(>p)$ \\
$K$ & ordering cost per order $(>0)$ \\
$h$ & unit holding cost per unit time $(h>0)$ \\
$\lambda$ & scale parameter of the demand function $(>0)$ \\
$\beta$ & elasticity parameter of the demand regarding the stock level $(0 \leq \beta<1)$ \\
$D(t)$ & demand rate at time $t, D(t)=\lambda[I(t)]^{\beta}$ \\
\hline
\end{tabular}

With this notation, the differential equation which defines the inventory level $I(t)$, for each $t \in[0, T)$, is given by

$$
\frac{d}{d t} I(t)=-\lambda[I(t)]^{\beta}
$$

with the initial condition $I(0+)=S$. The solution of the above equation is

$$
I(t)=\left(S^{1-\beta}-(1-\beta) \lambda t\right)^{1 /(1-\beta)}
$$

Moreover, as $I(T-)=s$, the length of the inventory cycle is evaluated as

$$
T=\frac{1}{(1-\beta) \lambda}\left(S^{1-\beta}-s^{1-\beta}\right) .
$$

Now, the relevant quantities in each inventory cycle can be evaluated as follows:

- $\quad$ Ordering cost to replenish the inventory $=K$;

- $\quad$ Purchasing cost of the items $=p(S-s)$;

- $\quad$ Holding cost $H(s, S)$ to keep items through the inventory cycle:

$$
H(s, S)=\int_{0}^{T} h I(t) d t=\frac{h}{\lambda(2-\beta)}\left(s^{2-\beta}-s^{2-\beta}\right)
$$


- $\quad$ Sales revenue of the items $=v(S-s)$.

The total cost in each inventory cycle is the sum of the purchasing cost, the ordering cost, and the holding cost. Then, the total cost per unit time $T C(s, S)$ is

$$
T C(s, S)=\frac{p(S-s)+K+H(s, S)}{T} .
$$

The profit per unit time $G(s, S)$ is given by

$$
G(s, S)=\frac{(v-p)(S-s)-K-H(s, S)}{T}
$$

and the return on investment $R(s, S)$ for the inventory model is the ratio between these two functions. That is,

$$
R(s, S)=\frac{G(s, S)}{T C(s, S)}=\frac{v}{p+r(s, S)}-1
$$

where

$$
r(s, S)=\frac{K+H(s, S)}{S-s}=\frac{K}{S-s}+\frac{h\left(S^{2-\beta}-s^{2-\beta}\right)}{\lambda(2-\beta)(S-s)}
$$

represents the average inventory cost per item (excluding the purchasing cost). Note that, as $r(s, S)>0$, then $-1<R(s, S)<v / p-1$.

As the goal of the model is the maximization of the return on investment $R(s, S)$, the mathematical problem is

$$
\max _{\substack{S>0 \\ 0 \leq s<S}} R(s, S)
$$

which is equivalent to

$$
\min _{\substack{S>0 \\ 0 \leq s<S}} r(s, S)
$$

This is an interesting issue for inventory managers: the maximization of the return on investment $R(s, S)$ is equivalent to the minimization of the average inventory cost per item $r(s, S)$.

As a consequence, the goal is different from the classical objectives for inventory models, which are either the inventory cost minimization or the profit maximization per unit time. Note that, as the cycle time $T$ is given by (3), the inventory cost per unit time is

$$
C(s, S)=\frac{K+H(s, S)}{T}=\frac{\lambda(1-\beta)}{S^{1-\beta}-s^{1-\beta}}\left(K+\frac{h\left(S^{2-\beta}-s^{2-\beta}\right)}{\lambda(2-\beta)}\right),
$$

and, from (3) and (6), the profit per unit time is

$$
G(s, S)=\frac{\lambda(1-\beta)}{S^{1-\beta}-s^{1-\beta}}\left((v-p)(S-s)-K-\frac{h\left(S^{2-\beta}-s^{2-\beta}\right)}{\lambda(2-\beta)}\right) .
$$

Furthermore, it is easily seen that, with constant demand rate (i.e., $\beta=0$ ), we have $C(s, S)=\lambda r(s, S)$ and $G(s, S)=\lambda(v-p-r(s, S))$. Then, minimizing the average inventory cost per item $r(s, S)$ is also equivalent to minimizing the inventory cost per unit time $C(s, S)$ and maximizing the profit per unit time $G(s, S)$.

Nevertheless, with an inventory level dependent demand rate (that is, $0<\beta<1$ ), this is not true. For this case, the problem of maximizing the function $G(s, S)$ was studied by Baker and Urban [8], and they proved that this function does not satisfy the usual quasi-concavity condition. Moreover, no closed expressions for the optimal solution could be obtained, and they only found an approximate solution using a non-linear programming algorithm with separable programming. The other two problems for the maximization 
of $R(s, S)$ and for the minimization of $C(s, S)$ are solved in this paper, obtaining closed expressions for the optimal solutions.

\section{Solution of the Model}

In order to solve the problem (10), we first prove that, for any order level $S$, the function $r_{S}(s)$ defined as $r_{S}(s)=r(s, S)$, with $r(s, S)$ given by (8) and $s \in[0, S)$, is strictly increasing and, therefore, the minimum value is obtained at the point $s^{*}(S)=0$.

Proposition 1. Given a fixed value $S>0$, the function $r_{S}(s)=r(s, S)$ with $s \in[0, S)$, is a strictly increasing function.

Proof. Please see the proof in Appendix A.

The next corollary is a consequence of the previous result:

Corollary 1. For any value of $S$, the function $r_{S}(s)$ reaches its minimum value at the point $s^{*}(S)=0$ and, therefore, $\min _{0 \leq s<S} r_{S}(s)=r(0, S)=\frac{K}{S}+\frac{h}{\lambda(2-\beta)} S^{1-\beta}$.

Proof. It follows directly from Proposition 1 and expression (8).

From these results, the solution of problem (10) can be obtained with the next theorem, which establishes the optimal policy that minimizes the average inventory cost per item $r(s, S)$.

Theorem 1. The minimum value for the function $r(s, S)$ given by (8) is reached at the point $\left(s^{*}, S^{*}\right)=\left(0, q^{*}\right)$, where

$$
q^{*}=\left(\frac{\lambda K(2-\beta)}{h(1-\beta)}\right)^{1 /(2-\beta)}
$$

and the optimal value is

$$
r^{*}=r\left(0, q^{*}\right)=\frac{(2-\beta) K}{(1-\beta) q^{*}}=\left(\frac{(2-\beta) K}{1-\beta}\right)^{(1-\beta) /(2-\beta)}\left(\frac{h}{\lambda}\right)^{1 /(2-\beta)} .
$$

Proof. Please see the proof in Appendix A.

Note that the solution (13) is a generalization of Harris-Wilson's formula because, for $\beta=0$, it leads to $S^{*}=q^{*}=\sqrt{2 \lambda K / h}$. Moreover, if $\beta=0$, the expression (14) leads to $r^{*}=2 K / q^{*}$ and, taking into account that $K / q^{*}$ is the optimal ordering cost per unit of item, it is clear that the optimal holding cost per item is equal to the optimal ordering cost per item. That is, in this case, we have a generalization of Harris' rule in EOQ models. Moreover, the next corollary provides a measure of sensitivity for the minimum inventory cost per item, which generalizes the similar expression for the $E O Q$ model.

Corollary 2. For any order level $S>0$, it satisfies that

$$
\frac{r(0, S)}{r^{*}}=\frac{1-\beta}{2-\beta}\left(\frac{S^{*}}{S}\right)+\frac{1}{2-\beta}\left(\frac{S}{S^{*}}\right)^{1-\beta},
$$

where $S^{*}$ and $r^{*}$ are given by (13) and (14), respectively.

Proof. It is easily seen from (8), (13), and (14).

All the previous results allow us to determine the optimal policy for the maximum ROI, which is given in the next theorem. 
Theorem 2. The return on investment $R(s, S)$ reaches the maximum value at the point $\left(s^{*}, S^{*}\right)=$ $\left(0, q^{*}\right)$, where $q^{*}$ is given by (13), and the optimal value is

$$
R^{*}=R\left(0, q^{*}\right)=\frac{v}{p+\frac{(2-\beta) K}{(1-\beta) q^{*}}}-1=\frac{(v-p)(1-\beta) q^{*}-(2-\beta) K}{p(1-\beta) q^{*}+(2-\beta) K} .
$$

Proof. It follows directly from Theorem 1 and the equivalence of the problems (9) and (10).

When the objective is to find the optimal policy with minimum inventory cost per unit time, the mathematical problem becomes this other

$$
\min _{\substack{S>0 \\ 0 \leq s<S}} C(s, S)
$$

where $C(s, S)$ is given by (11). In this case, the optimal solution can be obtained with the next result.

Proposition 2. Problem (16) reaches its solution at the point $\left(s_{1}^{*}, S_{1}^{*}\right)$, with $s_{1}^{*}=0$ and

$$
S_{1}^{*}=q_{1}^{*}=\left(\frac{\lambda K(1-\beta)(2-\beta)}{h}\right)^{1 /(2-\beta)} .
$$

Furthermore, the minimum cost per unit time is given by

$$
C^{*}=C\left(0, q_{1}^{*}\right)=\frac{\lambda K(1-\beta)(2-\beta)}{\left(q_{1}^{*}\right)^{1-\beta}}=h q_{1}^{*} .
$$

Proof. Please see the proof in Appendix A.

Theorem 1 and Proposition 2 prove that both the optimal solution of minimum cost per item and the optimal solution of minimum cost per unit time are obtained with the zero order point, and the known rule of not making a new order while stock is available is effective in both cases. Therefore, the order level must be equal to the lot size. Moreover, from (13) and (17), the ratio between the two solutions is

$$
\frac{q_{1}^{*}}{q^{*}}=(1-\beta)^{2 /(2-\beta)}<1,
$$

which means that, if $0<\beta<1$, the optimal lot size $q^{*}$ for the minimum cost per item is greater than the optimal lot size $q_{1}^{*}$ for the minimum cost per unit time. Obviously, if the demand rate is constant, i.e., $\beta=0$, both solutions are equal. Note that both solutions do not depend on the values $p$ and $v$ of the purchasing cost and the selling price. This is an interesting result for inventory managers because the optimal lot size does not change when these prices vary, as long as the objective is the maximization of the return on investment or the minimization of the inventory cost per unit of time.

It is also interesting for inventory managers to highlight the fact that the solution with maximum ROI is obtained with the zero order point, while the solution with maximum profit per unit time can lead to a non-zero order point. In addition, this last solution depends on the purchasing cost and the selling price, as Baker and Urban [8] proved.

From (4) and (13), we can ensure that

$$
K=(1-\beta) H\left(0, q^{*}\right) ;
$$

therefore, for the optimal solution with maximum ROI, the ordering cost is less than the holding cost. In a similar way, from (4) and (17), we have $H\left(0, q_{1}^{*}\right)=(1-\beta) K$, and, for the optimal solution of de minimum cost per unit time, the holding cost is less than the 
ordering cost. If the demand rate is constant, i.e., $\beta=0$, we have $q^{*}=q_{1}^{*}$ and $K=H\left(0, q^{*}\right)$, as the Harris' rule proposes for the basic EOQ model.

In addition, the expressions (14) and (15) can be used to ensure that the optimal return on investment is positive $\left(R^{*}>0\right)$ if, and only if, the selling price $v$ satisfies the condition

$$
v>p+r^{*}=p+\left(\frac{K(2-\beta)}{1-\beta}\right)^{(1-\beta) /(2-\beta)}\left(\frac{h}{\lambda}\right)^{1 /(2-\beta)} .
$$

Therefore, the initial parameters of the model define what the minimum selling price should be for the inventory to be profitable.

On the other hand, as the optimal order point is $s^{*}=0$, we can ensure that the inventory cycle time for the maximum ROI policy is

$$
T^{*}=\frac{\left(q^{*}\right)^{1-\beta}}{(1-\beta) \lambda} .
$$

From this expression, the inventory cost per unit time and the profit per unit time for the solution with the maximum return on investment can be evaluated as follows:

$$
C\left(0, q^{*}\right)=\frac{r^{*} q^{*}}{T^{*}}=\frac{\lambda K(2-\beta)}{\left(q^{*}\right)^{1-\beta}},
$$

and

$$
G\left(0, q^{*}\right)=\frac{(v-p) q^{*}-r^{*} q^{*}}{T^{*}}=\frac{(1-\beta)(v-p) \lambda q^{*}-\lambda K(2-\beta)}{\left(q^{*}\right)^{1-\beta}} .
$$

Similarly, for the solution of the minimum cost per unit time $q_{1}^{*}$, as the inventory cost in a cycle is $K+H\left(0, q_{1}^{*}\right)=K(2-\beta)$, the return on investment (ROI) and the profit per unit time can be evaluated as

$$
\begin{gathered}
R\left(0, q_{1}^{*}\right)=\frac{v}{p+K(2-\beta) / q_{1}^{*}}-1=\frac{(v-p) q_{1}^{*}-K(2-\beta)}{p q_{1}^{*}+K(2-\beta),} \\
G\left(0, q_{1}^{*}\right)=\frac{(1-\beta)(v-p) \lambda q_{1}^{*}-\lambda K(2-\beta)(1-\beta)}{\left(q_{1}^{*}\right)^{1-\beta}} .
\end{gathered}
$$

\section{Sensitivity Analysis}

In this section, we analyze how the optimal policy changes when the parameters of the model vary. To do this, the expressions (13) and (15) are used to calculate the partial derivatives of the optimal lot size $q^{*}$ and the maximum return on investment $R^{*}$ regarding the parameters of the inventory model. The practical issues deduced are given in the next two theorems.

Theorem 3. Let $q^{*}$ be the optimal lot size for the maximum ROI solution given by (13). Then:

(i) $q^{*}$ increases as the ordering cost $K$, or the scale parameter $\lambda$ of the demand rate, increase.

(ii) $q^{*}$ decreases as the unit holding cost $h$ increases.

(iii) If $\frac{\lambda K}{h}>\frac{\exp \left\{-(1-\beta)^{-1}\right\}}{1+(1-\beta)^{-1}}$ then $q^{*}$ increases as the elasticity parameter of the demand rate $\beta$ increases. In any other case, $q^{*}$ decreases as $\beta$ increases.

Proof. Please see the proof in Appendix A.

From the partial derivatives obtained in paragraphs (i) and (ii) of the previous proof, it is clear that

$$
\frac{\partial q^{*} / \partial K}{q^{*} / K}=-\frac{\partial q^{*} / \partial h}{q^{*} / h}=\frac{\partial q^{*} / \partial \lambda}{q^{*} / \lambda} .
$$


Then, the instantaneous relative changes in $q^{*}$ regarding the relative changes in the parameters $K, h$, or $\lambda$ are equal, except for the sign.

For the maximum return on investment, the results are given by the following theorem.

Theorem 4. Let $q^{*}$ be the optimal lot size given by (13) and $R^{*}$ the optimal value for the maximum ROI given by (15). Then:

(i) $R^{*}$ increases as the unit selling price $v$, or the scale parameter $\lambda$ of the demand rate, increase.

(ii) $R^{*}$ decreases as the unit purchasing cost $p$, or the ordering cost $K$, or the unit holding cost $h$, increase.

(iii) If $\frac{\lambda K}{h}>\frac{(1-\beta) \exp \{1\}}{2-\beta}, R^{*}$ increases as the elasticity parameter of the demand rate $\beta$ increases. In any other case, $R^{*}$ decreases as $\beta$ increases.

Proof. Please see the proof in Appendix A.

From the partial derivatives obtained in the previous proof, it is clear that

$$
-\left(\frac{1}{1-\beta}\right) \frac{\partial R^{*} / \partial K}{R^{*} / K}=-\frac{\partial R^{*} / \partial h}{R^{*} / h}=\frac{\partial R^{*} / \partial \lambda}{R^{*} / \lambda} .
$$

Then, the maximum return on investment $R^{*}$ is more sensitive to relative changes in $h$ or $\lambda$ than in $K$. In the basic $E O Q$ model, as $\beta=0$, the relative effect of the three parameters is the same, except sign. With respect to the unit purchasing cost $p$ and the unit selling price $v$, as $\frac{v}{p\left(1+R^{*}\right)}>1$ because of $R^{*}<v / p-1$, we have

$$
\frac{\partial R^{*} / \partial v}{R^{*} / v}=-\frac{v}{p\left(1+R^{*}\right)} \frac{\partial R^{*} / \partial p}{R^{*} / p},
$$

and, from a relative point of view, the maximum return on investment $R^{*}$ is more sensitive to relative changes in $v$ than in $p$, but with the opposite sign. However, if the inventory is profitable $\left(R^{*}>0\right)$, then the maximum return on investment is more sensitive to an absolute change in $p$ than to an equal change in $v$.

\section{Computational Results}

In this section, numerical examples are used to illustrate the proposed model and the solution methodology. In addition, the changes of the optimal solution regarding the parameters of the model are analyzed.

To compare the optimal solution here obtained with the solution for the maximum profit per unit time given by Baker and Urban [8], we start by using the same example included in their paper. Thus, the following parameters are considered: $K=10, p=10$, $v=20, h=0.5, \lambda=0.5$, and $\beta=0.4$.

From Theorem 2, the optimal order point is $s^{*}=0$, and, using the expression (13), the optimal order level is equal to the optimal lot size, with $q^{*}=S^{*}=7.78$. The maximum ROI given by the expression (15) leads to $R^{*}=0.4897$, i.e., with this optimal policy, the return on investment is $48.97 \%$. By using these values $s^{*}$ and $S^{*}$ in the expression (4), the holding cost per cycle can be evaluated as $H(0,7.78)=16.67$, and the minimum average inventory cost per item, given by the expression (14), is $r^{*}=3.43$. The optimal cycle time, given by the expression (22), is $T^{*}=11.42$, and the total cost per unit time, given by $(5)$, is $T C(0,7.78)=9.15$. In addition, note that the holding cost per cycle $H(0,7.78)=16.67$ is greater than the ordering $\operatorname{cost} K=10$, and the relation between them is $K=(1-\beta) H\left(0, q^{*}\right)$, as given by the expression (20). For this optimal solution, the inventory cost per unit time (excluding the purchasing cost) is evaluated with the expression (23) as $C\left(0, q^{*}\right)=2.34$. Similarly, the profit per unit time for this optimal solution is evaluated with the expression (24) and the obtained value is $G\left(0, q^{*}\right)=4.48$.

The solution with minimum cost per unit time has also been obtained by using Proposition 2, and the values are $s_{1}^{*}=0$ and $S_{1}^{*}=4.11$, with minimum cost per unit 
time $C^{*}=C(0,4.11)=2.06$. This value is less than the inventory cost per unit time obtained with the maximum ROI solution, which is $C\left(0, q^{*}\right)=2.34$. Instead, if we evaluate the average inventory cost per item for this solution, by using the expression (8), the obtained value is $r(0,4.11)=3.89$. This value is greater than the minimum inventory cost per item $r^{*}=3.43$. As a consequence, the ROI for this solution, evaluated with the expression (7), leads to $R(0,4.11)=0.4397$, which is less than the maximum return on investment $R^{*}=0.4897$. That is, the ROI with the solution of minimum cost per unit time is $5 \%$ lower than the maximum return on investment $R^{*}$. In addition, $S_{1}^{*}=q_{1}^{*}=4.11<$ $7.78=q^{*}$, and the relation between them is $q_{1}^{*} / q^{*}=(1-\beta)^{2 /(2-\beta)}$, as in (19).

For the problem of maximum profit per unit time, Baker and Urban [8] obtained an approximate solution using a non-linear programming algorithm with separable programming, which led to $S=5.0$ and $S=22.2$, with a profit per unit time equal to $G(5.0,22.2)=6.40$. For this example, the maximum value of the function $G(s, S)$ given by (12) has been calculated by using a numerical search procedure. The obtained solution was $s=3.40$ and $S=20.67$, with a profit per unit time equal to $G(3.40,20.67)=6.46$. Note that this value is greater than the profit per unit time provided with the solution of maximum ROI, i.e., $G\left(0, q^{*}\right)=4.48$. Instead, if we evaluate the return on investment of the solution for the maximum profit per unit time, using the expression (7), the obtained value is $R(3.40,20.67)=0.3399$, which is less than the maximum return on investment $R^{*}=0.4897$. That is, the ROI with the solution of maximum profit per unit time is $15 \%$ lower than the maximum return on investment $R^{*}$.

All these results show that the three solutions are different and they can be quite distant when the demand rate depends on the inventory level (i.e., $\beta>0$ ). This does not happen in basic EOQ models with constant demand rate (i.e., $\beta=0$ ). Table 3 lists the values obtained for the three optimal policies.

Table 3. Comparison of the three problems.

\begin{tabular}{lccc}
\hline & $\begin{array}{c}\text { Minimum Cost } \\
\text { Per Unit Time }\end{array}$ & $\begin{array}{c}\text { Maximum Profit } \\
\text { Per Unit Time }\end{array}$ & $\begin{array}{c}\text { Maximum } \\
\text { ROI }\end{array}$ \\
\hline Order level $S$ & 4.11 & 20.67 & 7.78 \\
Order point $s$ & 0 & 3.40 & 0 \\
Lot size $q$ & 4.11 & 17.27 & 7.78 \\
Cycle time $T$ & 7.78 & 13.57 & 11.42 \\
$H(s, S)$ & 6.00 & 75.08 & 16.67 \\
$T C(s, S)$ & 7.34 & 19.00 & 9.15 \\
$C(s, S)$ & 2.06 & 6.27 & 2.34 \\
$G(s, S)$ & 3.23 & 6.46 & 4.48 \\
$r(s, S)$ & 3.89 & 4.93 & 3.43 \\
$R(s, S)$ & $43.97 \%$ & $33.99 \%$ & $\mathbf{4 8 . 9 7 \%}$ \\
\hline
\end{tabular}

Figure 1 shows the functions $R(0, q)$ (right vertical axis) and $C(0, q)$ (left vertical axis) to compare the solutions of the two problems solved in this paper.

In order to confirm all the results given in the previous section with the sensitivity analysis, Theorems 1 and 2 have been used to determine the optimal lot size and the maximum ROI when each parameter is moved, keeping all the others fixed. Table 4 includes the obtained values for changes in each parameter between $-50 \%$ and $+50 \%$ with a step $10 \%$. 


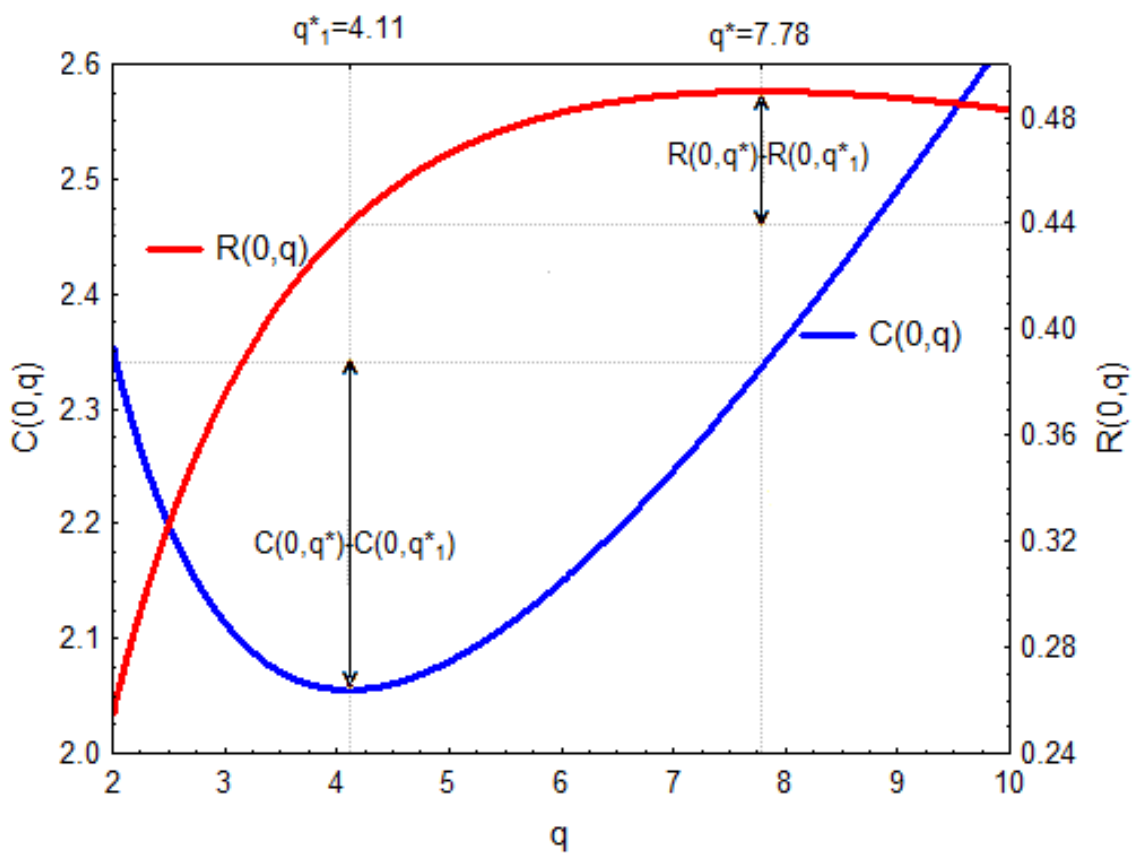

Figure 1. Return on investment (ROI) and inventory cost curves.

Table 4. Optimal ROI solution of the model with different values of the initial parameters.

\begin{tabular}{cccccccccccc}
\hline & $\boldsymbol{\Delta}$ & $\mathbf{- 5 0 \%}$ & $\mathbf{- 4 0 \%}$ & $\mathbf{- 3 0 \%}$ & $\mathbf{- 2 0 \%}$ & $\mathbf{- 1 0 \%}$ & $\mathbf{1 0 \%}$ & $\mathbf{2 0 \%}$ & $\mathbf{3 0 \%}$ & $\mathbf{4 0 \%}$ & $\mathbf{5 0 \%}$ \\
\hline$K=10$ & $R^{*}$ & 0.5821 & 0.5590 & 0.5388 & 0.5208 & 0.5046 & 0.4760 & 0.4633 & 0.4514 & 0.4403 & 0.4298 \\
& $q^{*}$ & 5.05 & 5.66 & 6.23 & 6.77 & 7.29 & 8.26 & 8.72 & 9.17 & 9.61 & 10.03 \\
\hline$h=0.5$ & $R^{*}$ & 0.6365 & 0.6014 & 0.5697 & 0.5409 & 0.5143 & 0.4667 & 0.4452 & 0.4249 & 0.4057 & 0.3876 \\
& $q^{*}$ & 12.01 & 10.71 & 9.73 & 8.95 & 8.31 & 7.33 & 6.95 & 6.61 & 6.31 & 6.04 \\
\hline$\lambda=0.5$ & $R^{*}$ & 0.3086 & 0.3592 & 0.4005 & 0.4349 & 0.4643 & 0.5120 & 0.5318 & 0.5495 & 0.5654 & 0.5799 \\
& $q^{*}$ & 5.05 & 5.66 & 6.23 & 6.77 & 7.29 & 8.26 & 8.72 & 9.17 & 9.61 & 10.03 \\
\hline$\beta=0.4$ & $R^{*}$ & 0.4296 & 0.4405 & 0.4519 & 0.4639 & 0.4765 & 0.5036 & 0.5183 & 0.5337 & 0.5500 & 0.5672 \\
& $q^{*}$ & 5.64 & 5.96 & 6.33 & 6.75 & 7.23 & 8.44 & 9.21 & 10.14 & 11.27 & 12.67 \\
\hline$v=20$ & $R^{*}$ & -0.2552 & -0.1062 & 0.0428 & 0.1918 & 0.3407 & 0.6387 & 0.7876 & 0.9366 & 1.0856 & 1.2345 \\
& $q^{*}$ & 7.78 & 7.78 & 7.78 & 7.78 & 7.78 & 7.78 & 7.78 & 7.78 & 7.78 & 7.78 \\
\hline$p=10$ & $R^{*}$ & 1.3737 & 1.1219 & 0.9184 & 0.7505 & 0.6096 & 0.3864 & 0.2965 & 0.2176 & 0.1477 & 0.0854 \\
& $q^{*}$ & 7.78 & 7.78 & 7.78 & 7.78 & 7.78 & 7.78 & 7.78 & 7.78 & 7.78 & 7.78 \\
\hline
\end{tabular}

To analyze the relative changes in the optimal solution regarding the relative changes in each parameter, keeping all the others fixed, several changes between $-50 \%$ and $+50 \%$ have been considered in each of the parameters. The optimal lot size $q^{*}$ and the maximum return on investment $R^{*}$ are shown in Figures 2 and 3.

Figure 2 shows that the optimal lot size does not depend on the parameters $v$ and $p$, and the changes in $q^{*}$ with respect to the parameters $K$ and $\lambda$ are equal. Positive increases in $\beta$ lead to a higher changes in the optimal lot size than the ones obtained with the other parameters. Nevertheless, if $\beta$ decreases, the changes in $q^{*}$ are similar to those obtained with $K$ or $\lambda$. These results are consistent with the statements in Theorem 3 and the equalities of the expression (27). 


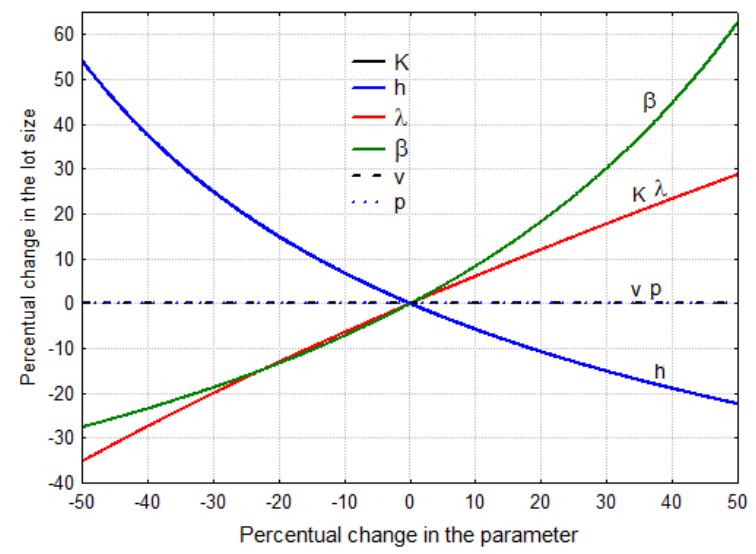

Figure 2. Percentual changes in $q^{*}$.

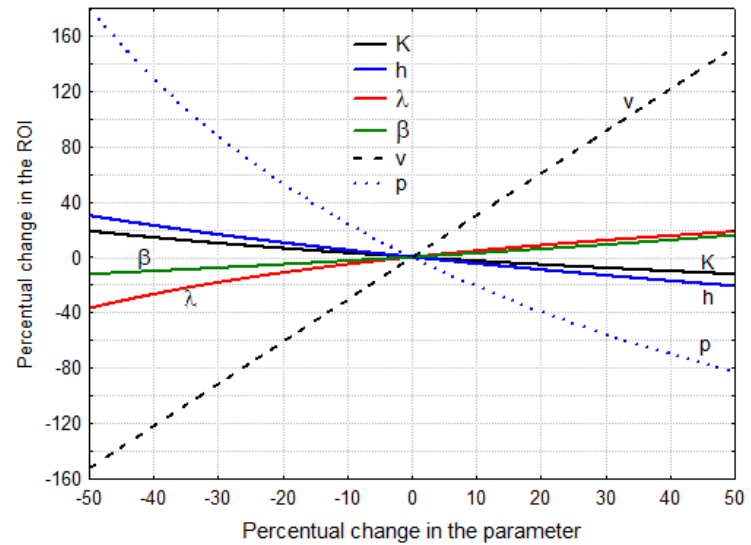

Figure 3. Percentual changes in $R^{*}$.

Figure 3 shows that the changes in the maximum return on investment $R^{*}$ are slightly larger with respect to $h$ than $K$. These results are also consistent with the statements in Theorem 4 and the equalities of the expression (28). The changes related to the parameters $v$ or $p$ are much greater than regarding the rest of the parameters.

\section{Conclusions and Managerial Insights}

This paper studies a deterministic inventory model with $(s, S)$ policy, focused on the maximization of the return on investment (ROI), instead of the minimization of the inventory cost, or the maximization of the profit per unit time.

The optimal solution shows that the inventory manager must wait for the stock to run out to replenishment the inventory (because the optimal order point is zero). Therefore, with the optimal policy, the order level $S$ is equal to the lot size $q$. The value for the optimal lot size is obtained in a closed-form. A proper balance between the ordering cost and the holding cost allows the maximum ROI policy to be identified.

In addition, we also give the optimal policy for the minimization of the inventory cost per unit time. For the two problems, the optimal policy does no change if the selling price or the unit purchasing cost vary. In addition, in the two problems, a new order should be required just when the stock is depleted. On the other hand, two differences are observed: the maximum ROI policy needs a greater optimal lot size and a holding cost greater than the ordering cost, while, in the solution of minimum cost per unit time, the opposite occurs.

The sensitivity analysis shows that an increase in the ordering cost $K$, or in the scale parameter of the demand rate $\lambda$, leads to an increase in the optimal lot size. Nevertheless, an increase in the unit holding cost per unit time $h$ leads to a decrease in the optimal lot size. The relative change in the optimal lot size regarding the relative changes in the parameters 
$K$ or $\lambda$ is the same, while, regarding the parameter $h$, it is the same but with the opposite sign. An increase in the elasticity parameter of the demand rate can lead to an increase or a decrease in the optimal lot size, depending on the values of the other parameters of the model.

With respect to the optimal ROI, the sensitivity analysis shows that an increase in the unit selling price $v$, or in the scale parameter $\lambda$ of the demand rate, leads to an increase in the optimal ROI. Nevertheless, an increase in any of the parameters $p, K$, or $h$ leads to a decrease in the optimal ROI. Analyzing the changes in a relative way, the maximum ROI is more sensitive to relative changes in the selling price $v$ than to relative changes in the purchasing cost $p$. In addition, it is more sensitive to relative changes in the scale parameter $\lambda$ of the demand rate than to relative changes in the unit holding cost $h$. The sensitivity regarding relative changes in the ordering $\operatorname{cost} K$, is greater than regarding the parameters $h$ or $\lambda$. Finally, an increase in the elasticity parameter of the demand rate leads to an increase or a decrease in the maximum ROI, depending on the values given for the other parameters of the inventory system.

The following managerial insights have been derived from the results:

- The maximum profitability solution (ROI solution) does not match the maximum profit per unit time solution in inventory models. So, managers should first select the proper objective for the inventory system.

- When the demand rate depends on the inventory level and the replenishment is instantaneous, managers should wait until the stock is depleted to replenish the inventory, as long as the goal is to obtain the maximum profitability. This is also true if the objective is the minimization of the inventory cost per unit time. However, the solution with maximum profit per unit time is obtained with a non-zero order point.

- In this context, maximizing profitability is equivalent to minimizing the average inventory cost per item (excluding the purchasing cost).

- If the demand depends on the inventory level, the optimal ROI is more sensitive to relative changes in the holding cost than to the same relative changes in the ordering cost.

- An absolute decrease in the unit purchasing cost leads to a larger increase in profitability than an equal increase in the unit selling price. However, for equal relative changes, the opposite occurs.

- The optimal inventory policy for ROI maximization does not change if the unit selling price, or the unit purchasing cost, vary. In addition, it is equally sensitive to relative changes in the ordering cost, the unit holding cost, or the scale parameter of the demand rate, except for the sign.

- The optimal lot size to obtain the maximum profitability is greater than the optimal lot size to obtain the minimum inventory cost per unit time.

The model considered in this paper can be extended incorporating perishable items, discounts in the unit purchasing cost or the unit selling price, or non-linear holding cost. The consideration of an integer demand could be a possible extension of the model. In addition, the use of the return on investment on the subject of stochastic inventory models needs to be developed. This would make it possible to formulate more realistic models.

Author Contributions: Conceptualization, V.P., L.A.S.-J., and J.S.; Methodology, V.P., L.A.S.-J., and J.S.; writing-review \& editing, V.P., L.A.S.-J., and J.S. All authors have read and agreed to the published version of the manuscript.

Funding: This work is partially supported by the Spanish Ministry of Science, Innovation and Universities through the research project MTM2017-84150-P, which is co-financed by the European Community under the European Regional Development Fund (ERDF).

Institutional Review Board Statement: Not applicable.

Informed Consent Statement: Not applicable. 
Acknowledgments: The authors wish to thank the editor and anonymous reviewers for the excellent services and constructive suggestions.

Conflicts of Interest: The authors declare no conflict of interest.

\section{Appendix A}

This appendix includes the proofs for some of the propositions and the theorems formulated in this paper.

Proof of Proposition 1. The function $r_{S}(s)$ is differentiable, and its derivative is given by

$$
\begin{aligned}
\frac{d}{d s} r_{S}(s) & =\frac{K}{(S-s)^{2}}+\frac{h\left(S^{2-\beta}-s^{1-\beta}((2-\beta) S-(1-\beta) s)\right)}{\lambda(2-\beta)(S-s)^{2}} \\
& =\frac{\lambda(2-\beta) K+h f_{S}(s)}{\lambda(2-\beta)(S-s)^{2}},
\end{aligned}
$$

where

$$
f_{S}(s)=S^{2-\beta}-(2-\beta) S s^{1-\beta}+(1-\beta) s^{2-\beta} .
$$

Taking into account that $f_{S}^{\prime}(s)=-(1-\beta)(2-\beta)(S-s) s^{-\beta}<0$ for all $s \in(0, S)$ and $\lim _{s \rightarrow S^{-}} f_{S}(s)=0$, we can ensure that the function $f_{S}(s)$ is positive.

Therefore, $\frac{d}{d s} r_{S}(s)>0$ and $r_{S}(s)$ is a strictly increasing function.

Proof of Theorem 1. From Corollary 1, it is sufficient to study the function $r(0, S)$ with $S \in(0, \infty)$, which satisfies that $\lim _{S \rightarrow 0^{+}} r(0, S)=\infty$ and $\lim _{S \rightarrow \infty} r(0, S)=\infty$. Moreover, $r(0, S)$ is a differentiable function with respect to $S$, and its derivative is

$$
\frac{d}{d S} r(0, S)=-\frac{K}{S^{2}}+\frac{h(1-\beta)}{\lambda(2-\beta)} S^{-\beta}=\frac{-\lambda(2-\beta) K+h(1-\beta) S^{2-\beta}}{\lambda(2-\beta) S^{2}} .
$$

Then, the only root for this derivative is the point $S^{*}$ given by (13). Moreover, $\frac{d}{d S} r(0, S)<0$ for $S \in\left(0, S^{*}\right)$ and $\frac{d}{d S} r(0, S)>0$ for $S \in\left(S^{*}, \infty\right)$. As a consequence, the optimal value is obtained for $S=S^{*}$. Finally, inserting the values $s^{*}$ and $S^{*}$ into expression (8), we obtain (14).

Proof of Proposition 2. The function $C(s, S)$ can be rewritten as

$$
C(s, S)=\lambda(1-\beta)\left(\frac{S-s}{S^{1-\beta}-s^{1-\beta}}\right) r(s, S),
$$

with $r(s, S)$ given by (8). Taking into account that $s<S$ and $0 \leq \beta<1$, it is easily seen that $s<S^{\beta} s^{1-\beta}$ and $S-s>S^{\beta}\left(S^{1-\beta}-s^{1-\beta}\right)$. Moreover, $r(s, S)>r(0, S)$ by Proposition (1). Thus, it is clear that, if $s>0$, then

$$
C(s, S)>\lambda(1-\beta) S^{\beta} r(0, S)=C(0, S) .
$$

Therefore, it is only necessary to solve the problem $\min _{S>0} C(0, S)$, with $C(0, S)=\lambda(1-$ $\beta)\left(\frac{K}{S^{1-\beta}}+\frac{h S}{\lambda(2-\beta)}\right)$. This function satisfies that $\lim _{S \rightarrow 0^{+}} C(0, S)=\lim _{S \rightarrow \infty} C(0, S)=$ $\infty$, and

$$
\frac{d}{d S} C(0, S)=\lambda(1-\beta)\left(-\frac{K(1-\beta)}{S^{2-\beta}}+\frac{h}{\lambda(2-\beta)}\right) .
$$


Then, the global minimum of this function is necessarily reached at the point $S$, which satisfies that $\frac{d}{d S} C(0, S)=0$. Now, it is clear that the only root $S_{1}^{*}$ of this equation is given by (17). Finally, inserting the values $S_{1}^{*}=q_{1}^{*}$ and $s_{1}^{*}=0$ into expression (11), we obtain

$$
\begin{aligned}
C^{*} & =C\left(0, q_{1}^{*}\right)=\lambda(1-\beta) q_{1}^{*}\left(\frac{K}{\left(q_{1}^{*}\right)^{2-\beta}}+\frac{h}{\lambda(2-\beta)}\right)=\lambda(1-\beta) q_{1}^{*}\left(\frac{K}{\left(q_{1}^{*}\right)^{2-\beta}}+\frac{K(1-\beta)}{\left(q_{1}^{*}\right)^{2-\beta}}\right) \\
& =\frac{\lambda(1-\beta)(2-\beta) K}{\left(q_{1}^{*}\right)^{1-\beta}}=h q_{1}^{*},
\end{aligned}
$$

and the proof is finished

\section{Proof of Theorem 3.}

(i) Taking into account that $(2-\beta) \ln \left(q^{*}\right)=\ln \lambda+\ln K-\ln h+\ln \left(\frac{2-\beta}{1-\beta}\right)$, and using logarithmic differentiation, we have $\frac{\partial q^{*}}{\partial K}=q^{*} \frac{\partial}{\partial K}\left[\frac{\ln K}{2-\beta}\right]=\frac{q^{*}}{(2-\beta) K}>0$ and $\frac{\partial q^{*}}{\partial \lambda}=q^{*} \frac{\partial}{\partial \lambda}\left[\frac{\ln \lambda}{2-\beta}\right]=\frac{q^{*}}{(2-\beta) \lambda}>0$.

(ii) It follows from $\frac{\partial q^{*}}{\partial h}=q^{*} \frac{\partial}{\partial h}\left[\frac{-\ln h}{2-\beta}\right]=-\frac{q^{*}}{(2-\beta) h}<0$.

(iii) In a similar way, we have

$$
\begin{aligned}
\frac{\partial q^{*}}{\partial \beta} & =q^{*} \frac{\partial}{\partial \beta}\left[\frac{\ln \lambda+\ln K-\ln h+\ln \left(\frac{2-\beta}{1-\beta}\right)}{2-\beta}\right]=q^{*}\left(\frac{\left(\frac{1-\beta}{2-\beta}\right)\left(\frac{1}{(1-\beta)^{2}}\right)}{2-\beta}+\frac{\ln \left(q^{*}\right)^{2-\beta}}{(2-\beta)^{2}}\right) \\
& =q^{*}\left(\frac{(1-\beta)^{-1}+\ln \left(q^{*}\right)^{2-\beta}}{(2-\beta)^{2}}\right) .
\end{aligned}
$$

Then, if $\frac{\lambda K}{h}>\frac{\exp \left\{-(1-\beta)^{-1}\right\}}{1+(1-\beta)^{-1}}$, as $1+(1-\beta)^{-1}=\frac{2-\beta}{1-\beta}$, we have

$$
\left(q^{*}\right)^{2-\beta}=\frac{\lambda K(2-\beta)}{h(1-\beta)}>\exp \left\{-(1-\beta)^{-1}\right\}
$$

which is equivalent to $\ln \left(q^{*}\right)^{2-\beta}>-(1-\beta)^{-1}$; therefore, $\frac{\partial q^{*}}{\partial \beta}>0$. As a consequence, $q^{*}$ increases as $\beta$ increases. In any other case, we have $\frac{\partial q^{*}}{\partial \beta} \leq 0$, and $q^{*}$ decreases as $\beta$ increases.

\section{Proof of Theorem 4.}

(i) Taking into account that $q^{*}$ does not depend on $v$ and $\frac{v}{1+R^{*}}-p=\frac{(2-\beta) K}{(1-\beta) q^{*}}$, the differentiation of this expression with respect to $v$ leads to

$$
\frac{1+R^{*}-v \frac{\partial R^{*}}{\partial v}}{\left(1+R^{*}\right)^{2}}=0
$$

Therefore, $\frac{\partial R^{*}}{\partial v}=\frac{1+R^{*}}{v}>0$, and $R^{*}$ increases as $v$ increases. 
In a similar way, by differentiation with respect to $\lambda$ and taking into account that $\frac{\partial q^{*}}{\partial \lambda}=\frac{q^{*}}{(2-\beta) \lambda}$, we have

$$
\frac{-v \frac{\partial R^{*}}{\partial \lambda}}{\left(1+R^{*}\right)^{2}}=\frac{-(2-\beta) K \frac{\partial q^{*}}{\partial \lambda}}{(1-\beta)\left(q^{*}\right)^{2}}=\frac{-K}{(1-\beta) \lambda q^{*}}
$$

Therefore, $\frac{\partial R^{*}}{\partial \lambda}=\frac{\left(1+R^{*}\right)^{2} K}{v(1-\beta) \lambda q^{*}}>0$, and $R^{*}$ increases as $\lambda$ increases.

(ii) Reasoning in the same way with $p, K$, and $h$, we obtain $\frac{\partial R^{*}}{\partial p}=-\frac{\left(1+R^{*}\right)^{2}}{v}<0$, $\frac{\partial R^{*}}{\partial K}=-\frac{\left(1+R^{*}\right)^{2}}{v q^{*}}<0$, and $\frac{\partial R^{*}}{\partial h}=-\frac{\left(1+R^{*}\right)^{2} K}{v(1-\beta) h q^{*}}<0$. Therefore, $R^{*}$ decreases as $p, K$, or $h$ increases.

(iii) With respect to the parameter $\beta$, we have

$$
\frac{\partial R^{*}}{\partial \beta}=\frac{\left(1+R^{*}\right)^{2} K}{v(1-\beta) q^{*}}\left(\ln q^{*}-\frac{1}{2-\beta}\right)
$$

Then, if $\frac{\lambda K}{h}>\frac{(1-\beta) \exp \{1\}}{2-\beta}$, as $\left(q^{*}\right)^{2-\beta}=\frac{\lambda K(2-\beta)}{h(1-\beta)}$, we have $\left(q^{*}\right)^{2-\beta}>\exp \{1\}$, and $(2-\beta) \ln q^{*}>1$. Therefore, $\frac{\partial R^{*}}{\partial \beta}>0$ and $R^{*}$ increases as $\beta$ increases. In any other case, we have $\frac{\partial R^{*}}{\partial \beta} \leq 0$, and $R^{*}$ decreases as $\beta$ increases.

\section{References}

1. Wolfe, H.B. A model for control of style merchandise. Ind. Manag. Rev. 1968, 9, 69-82.

2. Levin, R.I.; Mclaughlin, C.P.; Lamone, R.P.; Kottas, J.F. Production/Operations Management: Contemporary Policy for Managing Operating Systems; McGraw-Hill: New York, NY, USA, 1972.

3. Silver, E.A.; Peterson, R. Decision Systems for Inventory Management and Production Planning, 2nd ed.; John Wiley \& Sons: New York, NY, USA, 1985.

4. Larson, P.D.; DeMarais, R.A. Psychic stock: Retail inventory for stimulating demand. In Proceedings of the 1990 Academy of Marketing Science (AMS) Annual Conference, New Orleans, LA, 1990; Springer: Berlin, Germany, 2015; pp. 447-450.

5. Achabal, D.D.; McIntyre, S.; Smith, S.A. Maximizing Profits from Periodic Department Store Promotions. J. Retail. 1990, 66, 383.

6. Balakrishnan, A.; Pangburn, M.S.; Stavrulaki, E. "Stack them high, let 'em fly": Lot-sizing policies when inventories stimulate demand. Manag. Sci. 2004, 50, 630-644. [CrossRef]

7. Koschat, M.A. Store inventory can affect demand: Empirical evidence from magazine retailing. J. Retail. 2008, 84, 165-179. [CrossRef]

8. Baker, R.C.; Urban, T.L. A Deterministic Inventory System with an Inventory-Level-Dependent Demand Rate. J. Oper. Res. Soc. 1988, 39, 823-831. [CrossRef]

9. Padmanabhan, G.; Vrat, P. Inventory model with a mixture of backorders and lost sales. Int. J. Syst. Sci. 1990, 21, 1721-1726. [CrossRef]

10. Datta, T.K.; Pal, A.K. A Note on an Inventory Model with Inventory-level-dependent Demand Rate. J. Oper. Res. Soc. 1990, 41, 971-975. [CrossRef]

11. Urban, T.L. An Inventory Model with and Inventory-Level-Dependent Demand Rate and Relaxed Terminal Conditions. J. Oper Res. Soc. 1992, 43, 721-724. [CrossRef]

12. Pal, S.; Goswami, A.; Chaudhuri, K.S. A deterministic inventory model for deteriorating items with stock-dependent demand rate. Int. J. Prod. Econ. 1993, 32, 291-299. [CrossRef]

13. Giri, B.C.; Pal, S.; Goswami, A.; Chaudhuri, K.S. An inventory model for deteriorating items with stock-dependent demand rate. Eur. J. Oper. Res. 1996, 95, 604-610. [CrossRef]

14. Giri, B.C.; Chaudhuri, K.S. Deterministic models of perishable inventory with stock-dependent demand rate and nonlinear holding cost. Eur. J. Oper. Res. 1998, 105, 467-474. [CrossRef]

15. Datta, T.K.; Paul, K. An inventory system with stock-dependent, price-sensitive demand. Prod. Plan. Control 2001, 12, 13-20. [CrossRef] 
16. Ouyang, L.Y.; Hsieh, T.P.; Dye, C.Y.; Chang, H.C. An inventory model for deteriorating items with stock-dependent demand under the conditions of inflation and time-value of money. Eng. Econ. A J. Devoted Probl. Cap. Investig. 2003, 48, 52-68. [CrossRef]

17. Chang, C.T. Inventory models with stock-dependent demand and nonlinear holding costs for deteriorating items. Asia-Pac. J. Oper. Res. 2004, 21, 435-446. [CrossRef]

18. Pando, V.; García-Laguna, J.; San-José, L.A. Optimal policy for profit maximising in an EOQ model under non-linear holding cost and stock-dependent demand rate. Int. J. Syst. Sci. 2012, 43, 2160-2171. [CrossRef]

19. Pando, V.; San-José, L.A.; García-Laguna, J.; Sicilia, J. Maximizing profits in an inventory model with both demand rate and holding cost per unit time dependent on the stock level. Comput. Ind. Eng. 2012, 62, 599-608. [CrossRef]

20. Pando, V.; San-José, L.A.; García-Laguna, J.; Sicilia, J. An economic lot-size model with non-linear holding cost hinging on time and quantity. Int. J. Prod. Econ. 2013, 145, 294-303. [CrossRef]

21. Yang, C.-T. An inventory model with both stock-dependent demand rate and stock-dependent holding cost rate. Int. J. Prod. Econ. 2014, 155, 214-221. [CrossRef]

22. Annadurai, K.; Uthayakumar, R. Decaying inventory model with stock-dependent demand and shortages under two-level trade credit. Int. J. Adv. Manuf. Technol. 2015, 77, 525-543. [CrossRef]

23. Choudhury, K.D.; Karmakar, B.; Das, M.; Datta, T.K. An Inventory Model for Deteriorating Items with Stock-dependent Demand, Time-varying Holding Cost and Shortages. Opsearch 2015, 52, 55-74. [CrossRef]

24. Raymond, F.E. Quantity and Economy in Manufacture; McGraw-Hill: New York, NY, USA, 1931.

25. Schroeder, R.G.; Krishnan, R. Return on investment as a criterion for inventory models. Decis. Sci. 1976, 7, 697-704. [CrossRef]

26. Morse, W.J.; Scheeiner, J.H. Cost minimization, return on investment, residual income: Alternative criteria for inventory models. Acc. Bus. Res. 1979, 9, 320-324. [CrossRef]

27. Arcelus, F.J.; Srinivasan, G. A ROI-maximizing EOQ model under variable demand and markup rates. Eng. Costs Prod. Econ. 1985, 9, 113-117. [CrossRef]

28. Arcelus, F.J.; Srinivasan, G. Inventory policies under various optimizing criteria and variable markup rates. Manag. Sci. 1987, 33, 756-762. [CrossRef]

29. Giri, B.C. Optimal Pricing and Order-Up-To S Inventory Policy with expected Utility of the Present Value Criterion. Eng. Econ. J. Devoted Probl. Cap. Investig. 2014, 60, 231-244. [CrossRef]

30. Jordan, P.C. A comparative-analysis of the relative effectiveness of 4 dynamic lot-sizing techniques on return on investment. Decis. Sci. 1989, 20, 134-141. [CrossRef]

31. Rosenberg, D. Optimal price-inventory decisions profit vs. ROII. IIE Trans. 1991, 23, 17-22. [CrossRef]

32. Calderón-Rossell, J.R. Is the ROI a Good Indicator of the IRR?. Eng. Econ. J. Devoted Probl. Cap. Investig. 1992, 37, 315-340. [CrossRef]

33. Trietsch, D. Revisiting ROQ: EOQ For Company-wide ROI Maximization. J. Oper. Res. Soc. 1995, 46, 507-515. [CrossRef]

34. Otake, T.; Min, K.J.; Chen, C.-K. Inventory and investment in setup operations under return on investment maximization. Comput. Oper. Res. 1999, 26, 883-899. [CrossRef]

35. Otake, T.; Min, K.J. Inventory and investment in quality improvement under return on investment maximization. Comput. Oper. Res. 2001, 28, 997-1012. [CrossRef]

36. Li, J.; Min, K.J.; Otake, T.; Van Voorhis, T. Inventory and investment in setup and quality operations under return on investment maximization. Eur. J. Oper. Res. 2008, 185, 593-605. [CrossRef]

37. Protopappa-Sieke, M.; Seifert, R.W. Interrelating operational and financial performance measurements in inventory control. Eur. J. Oper. Res. 2010, 204, 439-448. [CrossRef]

38. Choi, T.-M.; Chiu, C.-H. Mean-downside-risk and mean-variance newsvendor models: Implications for sustainable fashion retailing. Int. J. Prod. Econ. 2012, 135, 552-560. [CrossRef]

39. Hidayat, Y.A.; Fauzi, M.R. Inventory Model for Deteriorating Items with Expired Time in Lost-Sales Probabilistic Demand. In Proceedings of the 2015 International Conference on Technology, Informatics, Management, Engineering \& Environment (TIME-E), Samosir Island, North Sumatra, Indonesia, 7-9 September 2015; pp. 40-46.

40. Wang, D.; Dimitrov, S.; Jian, L. Optimal inventory decisions for a risk-averse retailer when offering layaway. Eur. J. Oper. Res. 2020, 284, 108-120. [CrossRef]

41. Tao, L.; Liu, S.; Xie, N.; Javed, S.A. Optimal position of supply chain delivery window with risk-averse suppliers: A CVaR optimization approach. Int. J. Prod. Econ. 2021, 232, 107989. [CrossRef]

42. Ullah, M.; Asghar, I.; Zahid, M.; Omair, M.; AlArjani, A.; Sarkar, B. Ramification of remanufacturing in a sustainable three-echelon closed-loop supply chain management for returnable products. J. Clean. Prod. 2021, 290, 125609. [CrossRef]

43. Dey, B.K.; Pareek, S.; Tayyab, M.; Sarkar, B. Autonomation policy to control work-in-process inventory in a smart production system. Int. J. Prod. Res. 2021, 59, 1258-1280. [CrossRef]

44. Pando, V.; San-José, L.A.; Sicilia, J. Profitability ratio maximization in an inventory model with stock-dependent demand rate and non-linear holding cost. Appl. Math. Model. 2019, 66, 643-661. [CrossRef]

45. Ishfaq, R.; Bajwa, N. Profitability of online order fulfillment in multi-channel retailing. Eur. J. Oper. Res. 2019, 272, 1028-1040. [CrossRef]

46. Pando, V.; San-José, L.A.; Sicilia, J. A new approach to maximize the profit/cost ratio in a stock-dependent demand inventory model. Comput. Oper. Res. 2020, 120, 104940. [CrossRef] 